\title{
Paper
}

\section{Improved Signal Detection Techniques for QOSTBC System in Fast Fading Channel}

\author{
Jyoti P. Patra ${ }^{1}$ and Poonam Singh ${ }^{2}$ \\ ${ }^{1}$ Department of Electronics and Communication Engineering, Madanapalle Institute of Technology and Science, Madanapalle, India \\ ${ }^{2}$ Deartment of Electronics and Communication Engineering, National Institute of Technology, Rourkela, India
}

https://doi.org/10.26636/jtit.2020.138019

\begin{abstract}
Most existing quasi-orthogonal space time block coding (QO-STBC) schemes have been developed relying on the assumption that the channel is flat or remains static during the length of the code word symbol periods to achieve an optimal antenna diversity gain. However, in timeselective fading channels, this assumption does not hold and causes intertransmit-antenna-interferences (ITAI). Therefore, the simple pairwise maximum likelihood decoding scheme is not sufficient to recover original transmitted signals at the receiver side. To avoid the interferences, we have analyzed several signal detection schemes, namely zero forcing (ZF), two-step zero forcing (TS-ZF), minimum mean square error (MMSE), zero forcing - interference cancelation - decision feedback equalizer (ZF-IC-DFE) and minimum mean square error - interference cancelation - decision feedback equalizer (MMSE-IC-DFE). We have proposed two efficient iterative signal detection schemes, namely zero forcing - iterative interference cancelation - zero forcing - decision feedback equalization (ZF-IIC-ZF-DFE) and minimum mean square error - parallel interference cancelation - zero forcing - decision feedback equalization (MMSE-IIC-ZF-DFE). The simulation results show that these two proposed detection schemes significantly outperform all conventional methods for QOSTBC system over time selective channel.
\end{abstract}

Keywords-ITAI, QOSTBC, signal detection, time selective channel.

\section{Introduction}

Next generation wireless communication systems demand high link reliability and high data transmission rates, without sacrificing extra power or bandwidth due to the application of high quality audio and video signal in a highly mobile environment. Deployment of multiple antennas at the base or mobile station - a solution commonly known as multiple-input-multiple-output (MIMO) system, is capable of meeting these requirements, since it offers increases in data throughput and link reliability, without the need for additional bandwidth or transmit power compared to the traditional single-input-single-output (SISO) system [1]-[4]. In recent years, the transmit diversity schemes have been attracting much more attention after Alamouti proposed, in [5], an elegant space-time block code (STBC) for two transmit antennas and a single receive antenna, as such a solution achieves full diversity with low complexity linear ML decoding. The STBC scheme provides diversity with simple decoding and does not require extra power or bandwidth. Hence, it is adopted in almost all recent wireless standards including IEEE 802.11 ac Wi-Fi [6], [7], 3GPP LTE/LTE Advanced [8], [9], DVB-terrestrial 2nd generation (T2) [10], DVB-next generation handheld (NSH) [11], and many more applications.

However, it is shown in [12], [13] that the OSTBC schemes cannot provide both full rate and full diversity simultaneously for more than two transmit antennas. In order to achieve full data rate, several quasi-orthogonal STBC (QOSTBC) schemes have been introduced [14]-[16]. The QOSTBCs employ more than two transmit antennas which rely on pairwise-ML decoding to achieve the full rate, but with a reduction in diversity gain. The pairwise-ML method requires more computational complexity, as it has to perform joint ML decoding to recover two pairs of data signals separately, unlike OSTBC which only requires the linear ML method. Therefore, various methods have been proposed to simplify the pairwise-ML decoding scheme [17]-[20]. A linear ML decoding algorithm based on $\mathrm{QR}$ decomposition of the channel matrix was proposed in [17]. In [20], a conditional maximum-likelihood (ML) based detection scheme was proposed to reduce the decoding complexity of the QOSTBC system.

Most of the existing QOSTBC schemes that have been developed rely on the assumption that the channel remains quasi-static during the length of the code word symbol periods. Therefore, they are applicable only to time invariant flat fading channels. However, in practice, the channel is time-selective due to the movement of high speed vehicles. In such cases, the quasi-static assumption does not hold and causes severe performance degradation due to intertransmit-antenna-interferences (ITAI) [21]-[23] in addition to irreducible error floor from inter-symbol-interference (ISI) effects (due to partial diversity) [21]-[23]. Hence, the simple pairwise-ML method [15] is not sufficient to recover the original transmitted signal at the receiver side. 
To improve the performance of the system, a two-step ZF (TS-ZF) detector was proposed in [24], eliminating the interferences. This method comprises two steps. In the first step, a transform matrix is multiplied with the channel matrix to get a diagonal matrix, and simple least square detection is then performed to detect the transmitted signals. In addition to this linear method, non-linear methods have been developed as well, such as PIC [25] and SIC [26], to recover the transmitted signal for STBC systems with the number of transmit antennas greater than two. In [22], [23], the authors propose two detection schemes, namely zero forcing - interference cancelation - decision feedback equalization (ZF-IC-DFE) and minimum mean square error - interference cancelation - decision feedback equalization (MMSE-IC-DFE). These detection techniques estimate the initial signal using either ZF or MMSE methods followed by interference cancelation, to obtain the ITAI-free received signal. Finally, the ITAI-free signal is processed by an LDL factorization-based DFE scheme to obtain the desired data signal. As the DFE receiver recursively obtains the transmitted data signal in the reverse order, these methods may suffer from the error propagation problem if the initial signal does not contain reliable information.

In this paper, we propose two efficient iterative signal detection schemes to improve performance of the QOSTBC system. Initially, either the ZF or the MMSE method is used to estimate the transmitted signal. Then, by using the estimated signal originating from the ZF/MMSE method, the interferences are canceled iteratively, followed by the ZF-SIC method and soft decision. We call the proposed scheme zero forcing iterative - zero forcing - successive interference cancelation (ZI-ZF-SIC), or minimum mean square error iterative - zero forcing - successive interference cancelation (MI-ZF- SIC), depending upon whether the initial signal is estimated by using the ZF or the MMSE method.

The symbols associated with matrices and vectors are denoted in bold upper and lower-case letters, respectively. The notation $(.)^{H},(.)^{-1}$ denotes the Hermitian; $\operatorname{Re}($.$) stands for$ real part; $\operatorname{tr}($.$) denotes the trace operation; \|.\|^{2}$ denotes the Euclidean norm; $E[$.$] denotes the expectation operation; C_{M}$ denotes the constellation point; $\Pi$ hard decision function, $J_{0}($.$) zeroth order Bessel function.$

The rest of the paper is organized as follows. In Section 2, the system model for the QO-STBC is discussed. Various conventional detection methods are presented in Section 3. Section 4 describes the proposed detection methods. In Section 5, the performance of these signal detection methods is compared based on the bit error rate over various mobile velocities. Finally, Section 6 concludes the paper.

\section{QOSTBC System Model}

We consider the QOSTBC scheme proposed in [15] with 4 transmit antennas (4Tx) and one receive antenna (1Rx). Four complex signals $x_{1}, x_{2}, x_{3}$, and $x_{4}$ are first grouped

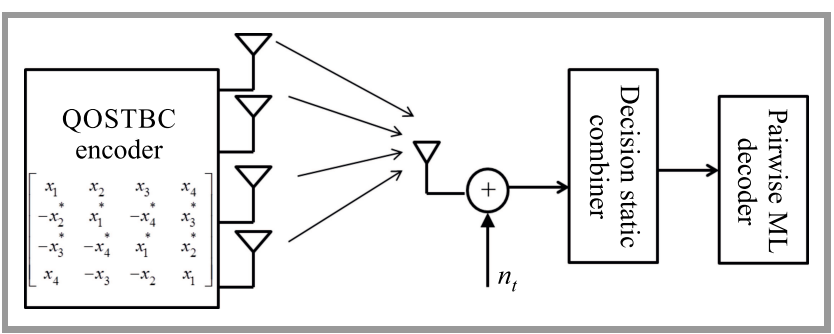

Fig. 1. Block diagram of QOSTBC system.

together and then passed through a QOSTBC encoder. The encoded transmitted signal matrix of the QOSTBC scheme can be written as:

$$
\mathbf{S}=\left[x_{i l}\right]=\left[\begin{array}{cccc}
x_{1} & x_{2} & x_{3} & x_{4} \\
-x_{2}^{*} & x_{1}^{*} & -x_{4}^{*} & x_{3}^{*} \\
-x_{3}^{*} & -x_{4}^{*} & x_{1}^{*} & x_{2}^{*} \\
x_{4} & -x_{3} & -x_{2} & x_{1}
\end{array}\right]
$$

where $x_{i l}$ denotes the signal transmitted from the $i$-th transmitted antenna at $l$-th time slot and $i, l \in\{1,2,3,4\}$. The received signal vector $\boldsymbol{r}$, after passing the encoded signal through the channel, can be expressed as:

$$
r=\mathbf{H} \boldsymbol{x}+\boldsymbol{n}
$$

where $\boldsymbol{r}=\left[\begin{array}{llll}r_{1} & r_{2}^{*} & r_{3}^{*} & r_{4}\end{array}\right]^{T}, \boldsymbol{x}=\left[\begin{array}{llll}x_{1} & x_{2} & x_{3} & x_{4}\end{array}\right]^{T}$ and $\boldsymbol{n}=\left[\begin{array}{llll}n_{1} & n_{2}^{*} & n_{3}^{*} & n_{4}\end{array}\right]^{T}$ where $n_{l}$ is the additive white Gaussian noise (AWGN) with zero mean and $\sigma_{n}^{2}$ variance during the $l$ th symbol period. The channel matrix $(\mathbf{H})$ is:

$$
\mathbf{H}=\left[\begin{array}{cccc}
h_{1}(1) & h_{2}(1) & h_{3}(1) & h_{4}(1) \\
h_{2}(2)^{*} & -h_{1}(2)^{*} & h_{4}(2)^{*} & -h_{3}(2)^{*} \\
h_{3}(3)^{*} & -h_{4}(3)^{*} & -h_{1}(3)^{*} & -h_{2}(3)^{*} \\
h_{4}(4) & -h_{3}(4) & -h_{2}(4) & h_{1}(4)
\end{array}\right] .
$$

Here, we assume each channel undergoes time selective flat fading generated from the Jakes sum-of-sinusoidal (SOS) [27] model with zero mean and autocorrelation $R_{h}(m)=E\left[h_{i}(l) h_{i}(l+m)^{*}\right]=\sigma_{h}^{2} \alpha_{m}$ where $\sigma_{h}^{2}$ denotes the power of path gain and we normalize the power of path gain to unity. According to the Jakes model, $\alpha_{m}=J_{0}\left(2 \pi f_{d} T_{s} m\right)$. Let us assume the channel is time invariant flat fading, i.e. $h_{i}=h_{i}(l)=h_{i}(l+m)$ for $m=1,2,3$. In such a scenario, the pairwise maximum likelihood (ML) method proposed in [15] is applied to separate the transmitted signals. This can be performed in two steps. In the first step, the matched filter operation is performed followed by a joint decoding of two symbol pairs using the ML method. The matched filter operation can be performed by multiplying $\mathbf{H}^{H}$ on both sides of Eq. (2), i.e.:

$$
\boldsymbol{r}^{\prime}=\mathbf{H}^{H} \mathbf{H} \boldsymbol{x}+\mathbf{H}^{H} \boldsymbol{n}=\Theta \boldsymbol{x}+\boldsymbol{n}^{\prime}
$$

where $\Theta$ is the Gramian matrix of the QOSTBC system and is given by: 


$$
\Theta=\mathbf{H}^{H} \mathbf{H}=\left[\begin{array}{cccc}
a & 0 & 0 & b \\
0 & a & -b & 0 \\
0 & -b & a & 0 \\
b & 0 & 0 & a
\end{array}\right]
$$

where $a=\sum_{i=1}^{4}\left|h_{i}\right|^{2}, b=2 \operatorname{Re}\left(h_{1} h_{4}{ }^{*}-h_{2} h_{3}{ }^{*}\right)$ are the diversity gain and irreducible ISI, respectively. The pairwiseML method separates the transmitted signal into the sum of two independent terms $f\left(x_{1}, x_{4}\right)+f\left(x_{2}, x_{3}\right)$ :

$$
\hat{x}=\underset{x \subset C_{M}^{2}}{\arg \min }\left\|\boldsymbol{r}^{\prime}-\Theta \boldsymbol{x}\right\|
$$

Thus, by applying the pairwise-ML method, the transmitted signal may be perfectly recovered from the mixed transmitted signals at the receiver side.

However, when the channel is time selective, the quasistatic assumptions does not hold good, i.e. $h_{i}(l) \neq h_{i}(l+m)$. Hence, $\mathbf{H}^{H} \mathbf{H}$ is no longer a quasi-orthogonal matrix and is given by:

$$
\begin{gathered}
\Theta=\mathbf{H}^{H} \mathbf{H}=\underbrace{\left[\begin{array}{cccc}
a_{1} & \Lambda_{1} & \Lambda_{2} & \beta \\
\Lambda_{1}^{*} & a_{2} & \partial & \Lambda_{3} \\
\Lambda_{2}^{*} & \partial^{*} & a_{3} & \Lambda_{4} \\
\beta^{*} & \Lambda_{3}^{*} & \Lambda_{4}^{*} & a_{4}
\end{array}\right]}_{\Theta} \\
=\underbrace{\left[\begin{array}{cccc}
a_{1} & 0 & 0 & \beta \\
0 & a_{2} & \partial & 0 \\
0 & \partial^{*} & a_{3} & 0 \\
\beta^{*} & 0 & 0 & a_{4}
\end{array}\right]}_{\Theta_{D}+\Theta_{A D}=\Theta_{S}}+\underbrace{\left[\begin{array}{cccc}
0 & \Lambda_{1} & \Lambda_{2} & 0 \\
\Lambda_{1}^{*} & 0 & 0 & \Lambda_{3} \\
\Lambda_{2}^{*} & 0 & 0 & \Lambda_{4} \\
0 & \Lambda_{3}^{*} & \Lambda_{4}^{*} & 0
\end{array}\right]}_{\Theta_{N D}},
\end{gathered}
$$

where $a_{i}$ denotes the desired diversity gain terms placed at the diagonal entry of the Gram matrix given as $\Theta_{D}$. Parameters $\beta$ and $\delta$ and their corresponding conjugates denote the ISI effect and are located on the anti-diagonal Gramian matrix, i.e. $\Theta_{A D}$. Parameter $\Lambda_{i}$ and its corresponding conjugate are the ITAI effects that occur due to the time-selective nature of the channel and are presented on the non-diagonal entry of the matrix, i.e. $\Theta_{N D}$. As the off-diagonal entries are not zeros, the pairwise-ML method cannot be sufficient to recover the original transmitted signal, as the likelihood function can no longer be decomposed into the sum of two pair-wise functions. Hence, we have analyzed various signal detection methods and also proposed two iterative methods to improve the performance of the system by canceling these interferences.

\section{Conventional Signal Detection Method}

Here, we describe different conventional detection methods, namely TS-ZF, ZF-IC-DFE and MMSE-IC-DFE for the QO-STBC system over a time selective flat fading channel.

\subsection{TS-ZF Method}

The two-step zero forcing (TS-ZF) method is proposed in [24] to detect the QOSTBC signal. As $\mathbf{H}^{H} \mathbf{H}$ does not give a quasi-orthogonal matrix, a transform matrix $\Omega$ is multiplied instead of $\mathbf{H}^{H}$ and is:

$$
\Omega x=\Omega H x+\Omega n=D x+n^{\prime},
$$

where $\mathbf{D}=\operatorname{diag}\left(\phi_{1}, \phi_{2}, \phi_{3}, \phi_{4}\right)$ and $\phi_{i}$ are the diagonal entry of the $\Omega \mathbf{H}$ matrix. Then, we apply the simple LS detection scheme to estimate the transmitted signal:

$$
\hat{x}_{i}=\underset{x_{i} \in C_{M}}{\arg \min }\left\|[\Omega]_{i} \boldsymbol{r}-\phi_{i} x_{i}\right\|^{2},
$$

where $[\Omega]_{i}$ denotes the $i$-th row of $\Omega$. The TS-ZF performs better than the pairwise-ML method, as it forces the two interference signals, i.e. ISI and ITAI to zero.

\subsection{ZF-IC-DFE Method}

Zero forcing - interference cancelation - decision feedback equalization (ZF-IC-DFE) was proposed in [23]. This method initially obtains the data signal using the $\mathrm{ZF}$ method [28], as:

$$
\hat{x}=\left[H^{H} \mathbf{H}\right]^{-1} H^{H} \boldsymbol{r},
$$

where $\mathbf{H}$ is the channel matrix of size $N_{T} \times N_{T}$ and $\boldsymbol{r}$ is the received signal vector of size $N_{T} \times 1$. Then, this initial data signal from the $\mathrm{ZF}$ method is used to estimate the ITAI signal and then cancel its contributions to obtain the ITAI-free received signal as:

$$
\boldsymbol{r}_{I Z}=\boldsymbol{r}^{\prime}-\Theta_{N D} \hat{x}=\Theta_{S} \hat{x}+\boldsymbol{n}^{\prime},
$$

where $\boldsymbol{r}^{\prime}=\mathbf{H}^{H} \boldsymbol{r}$ is the matched filtered output of the received signal. $\Theta_{N D}$ is the off-diagonal entry of the $\Theta$ matrix and contains the ITAI signal. The $\Theta_{N D}$ matrix is obtained by subtracting the diagonal and anti-diagonal of the Gramian matrix and is given by:

$$
\Theta_{N D}=\Theta-\left(\Theta_{D}+\Theta_{A D}\right) .
$$

Applying the Cholesky decomposition to the $\Theta_{S}$ matrix, we have:

$$
\Theta_{S}=\Theta_{D}+\Theta_{A D}=\mathbf{L}^{H} \mathbf{D} \mathbf{L},
$$

where $\mathbf{L}$ is an upper triangular matrix of size $N_{T} \times N_{T}$ with diagonal entries of value one, and $\mathbf{D}$ is a diagonal matrix of size $N_{T} \times N_{T}$. The DFE receiver can be obtained by pre-multiplying $\mathbf{D}^{-1} \mathbf{L}^{-H}$ with the received signal vector $\boldsymbol{r}$ :

$$
\boldsymbol{r}_{D}=\mathbf{D}^{-1} \mathbf{L}^{-\mathbf{H}} \boldsymbol{r}_{I Z}=\mathbf{L} x+\mathbf{D}^{-1} \mathbf{L}^{-H} \boldsymbol{n}^{\prime},
$$

where $\boldsymbol{r}_{D}$ is the output of DFE receiver which recursively obtains the transmitted data signal in the reverse order. Parameter $\mathbf{L}$ is an upper triangular matrix and is:

$$
\mathbf{L}=\left[\begin{array}{cccc}
1 & 0 & 0 & \Delta_{1} \\
0 & 1 & \Delta_{2} & 0 \\
0 & 0 & 1 & 0 \\
0 & 0 & 0 & 1
\end{array}\right]
$$


where $\Delta_{1}$ and $\Delta_{2}$ are coefficients of the feedback filter. The filter obtains $x_{3}$ and $x_{4}$ separately and then this information is used to help make the decision about $x_{1}$ and $x_{2}$.

\subsection{MMSE-IC-DFE Method}

The MMSE-IC-DFE works similarly to the previously mentioned ZF-IC-DFE method. It employs the MMSE method [28] in the first step instead of the ZF method to estimate the initial transmitted signal:

$$
\hat{x}=\left[\mathbf{H}^{H} \mathbf{H}+\sigma_{n}{ }^{2} \mathbf{I}_{N_{T}}\right]^{-1} \mathbf{H}^{H} \boldsymbol{r},
$$

where $\sigma_{n}^{2}$ is the noise variance and $\mathbf{I}_{N_{T}}$ is the identity matrix. The remaining two steps, i.e. interference cancellation and Cholesky decomposition based on DFE, are the same as in the previously mentioned ZF-IC-DFE detection method. Both ZF-IC-DFE and MMSE-IC-DFE outperform their corresponding linear detection counterparts, i.e. ZF and MMSE, because they apply interference cancelation and DFE to further improve the system's performance. However, the performance of these detection methods is not satisfactory due to the propagation of a less reliable transmitted signal to estimate the other transmitted signals. We have proposed two iterative signal detection methods which provide more reliable information signals and are described in the next section.

\section{Proposed Signal Detection Method}

We have proposed two iterative detection schemes, namely ZF-IIC-ZF-DFE and MMSE-IIC-ZF-DFE to cancel ISI and ITAI effects. In the first step, either the ZF or the MMSE method is used to estimate the initial signal and by using these estimated signal, ITAI effects are canceled iteratively. Then, by applying the ITAI-free received signal, ISI effects are canceled by adopting the ZF-DFE method. Finally, the ZF-DFE output signal is fed back to the ITAI canceler for the next iteration.

\subsection{ZF-IIC-ZF-DFE Method}

The zero forcing - iterative interference cancelation - zero forcing - decision feedback equalization (ZF-IIC-ZF-DFE) method uses the $\mathrm{ZF}$ method to estimate the initial data symbol. Then, by using the estimated signal coming from the ZF output, ITAI signals are generated and subtracted from the received signal iteratively. The ITAI-free received signal is processed by the ZF-DFE method to obtain a more refined data signal and is fed back for the next iteration. The description of ZF-IIC-ZF-DFE is given below.

Step 1: Set iteration number $k=0$ and obtain $\boldsymbol{x}^{(0)}$ from ZF method

$$
\boldsymbol{x}^{(0)}=\hat{x}
$$

Step 2: Estimate and cancel the ITAI signal iteratively (for $k=0,1, I-1)$ :

$$
\boldsymbol{r}_{\text {off_ITAI }}^{(k)}=\boldsymbol{r}^{\prime}-\Theta_{N D} \hat{x}^{(k-1)}
$$

where $\boldsymbol{r}_{o f f \_I T A I}^{(k)}$ is the ITAI free received signal in the $k$ th iteration and $I$ denotes predefined number of iterations.

Step 3: Separate $\boldsymbol{r}_{\text {off_ITAI }}^{(k)}$ into two symbol pairs $\left(x_{1}^{(k)}, x_{4}^{(k)}\right)$ and $\left(x_{2}^{(k)}, x_{3}^{(k)}\right)$ :

$$
\begin{aligned}
& {\left[\begin{array}{c}
r_{1, \text { off_ITAI }}^{(k)} \\
r_{4, \text { off_ITAI }}^{(k)}
\end{array}\right]=\left[\begin{array}{ll}
a_{1} & \beta \\
\beta^{*} & a_{4}
\end{array}\right]\left[\begin{array}{c}
x_{1}^{(k)} \\
x_{4}^{(k)}
\end{array}\right]+\left[\begin{array}{l}
n_{1}^{\prime} \\
n_{4}^{\prime}
\end{array}\right],} \\
& {\left[\begin{array}{c}
r_{2, \text { off_ITAI }}^{(k)} \\
r_{3, \text { off }(k) \text { ITAI }}
\end{array}\right]=\left[\begin{array}{cc}
a_{2} & \partial \\
\partial^{*} & a_{3}
\end{array}\right]\left[\begin{array}{c}
x_{2}^{(k)} \\
x_{3}^{(k)}
\end{array}\right]+\left[\begin{array}{c}
n_{2}^{\prime} \\
n_{3}^{\prime}
\end{array}\right] .}
\end{aligned}
$$

Step 4: Apply ZF method to obtain $\hat{x}_{1}$ and $\hat{x}_{4}$ signals which is given by:

$$
\begin{aligned}
& {\left[\begin{array}{c}
\tilde{x}_{1}^{(k)} \\
\tilde{x}_{4}^{(k)}
\end{array}\right]=\left[\begin{array}{ll}
a_{1} & \beta \\
\beta^{*} & a_{4}
\end{array}\right]^{-1}\left[\begin{array}{c}
r_{1, \text { off ITAI }}^{(k)} \\
r_{4, \text { off }(k) A I}
\end{array}\right]} \\
& =\frac{1}{\varphi}\left[\begin{array}{cc}
a_{4} & -\beta \\
-\beta^{*} & a_{1}
\end{array}\right]\left[\begin{array}{c}
r_{1, \text { off } I T A I}^{(k)} \\
r_{4, \text { off } \mathrm{k} \text { ITAI }}
\end{array}\right],
\end{aligned}
$$

where $\varphi=-\left(a_{1} a_{4}+|\beta|^{2}\right)$ is a scalar quantity.

Step 5: Perform DFE method to obtain refined $\hat{x}_{1}$ and $\hat{x}_{4}$ and feedback for next iteration:

$$
\begin{aligned}
& \hat{x}_{1}^{(k)}=\Pi\left(\frac{r_{1, \text { off_ITAI }}^{(k)}-\beta \tilde{x}_{4}^{(k)}}{a_{1} x_{1}^{(k)}}\right), \\
& \hat{x}_{4}^{(k)}=\Pi\left(\frac{r_{4, o f f \_I T A I}^{(k)}-\beta^{*} \tilde{x}_{1}^{(k)}}{a_{4} x_{4}^{(k)}}\right) .
\end{aligned}
$$

It is noted that, as the number of iteration increases, ITAI effects reduce and quality of the transmitted signal improves progressively. After a few iterations (I), ITAI effects are almost completely canceled out and we obtain the desired estimated data signal which is free of both ITAI and ISI effects.

\subsection{MMSE-IIC-ZF-DFE Method}

Performance of the previously proposed ZF-IIC-ZF-DFE method depends on the initial estimate of the signal, thus it is prone to error floor, as it utilizes the ZF method to obtain the initial signal. Therefore, performance can be improved further by applying the MMSE method as given in Eq. (16) instead of the ZF method to estimate the initial signal. This method is referred to as MMSE-IIC-ZF-DFE. The rest of the operating principle of this method is similar to that of ZF-IIC-ZF-DFE method. 


\section{Simulation Results and Discussions}

The performance comparison of various detection methods which include pairwise-ML, ZF, TS-ZF, MMSE, ZF-ICDFE, MMSE-IC-DFE, ZF-IIC-ZF-DFE and MMSE-IICZF-DFE is conducted based on BER for QOSTBC over a time selective flat fading channel. The Jakes sum of sinusoidal (SOS) model is adopted to construct the timeselective fading environment. A perfect channel information state is assumed at the receiver side. The simulation parameters used for simulations are presented in Table 1. For the QOSTBC system, we consider $4 T_{x}$ and a single receiver antenna. The signal-to-noise ratio at the receiver is defined as $\mathrm{SNR}=\left(N_{T} \sigma_{h}^{2} E_{S}\right) / \sigma_{n}^{2}=4 E_{S} / \sigma_{n}^{2}$ since $N_{T}=4, \sigma_{h}^{2}=1$.

Table 1

Simulation parameters

\begin{tabular}{|l|c|c|}
\hline \multicolumn{1}{|c|}{ Parameter } & \multicolumn{2}{c|}{ Value } \\
\hline \hline Antenna configurations & \multicolumn{2}{|c|}{$4 \mathrm{TX}, 1 \mathrm{RX}$} \\
\hline Channel model & \multicolumn{2}{|c|}{ Jakes model } \\
\hline Carrier frequency & \multicolumn{2}{|c|}{$2 \mathrm{GHz}$} \\
\hline Symbol duration & \multicolumn{2}{|c|}{$256 /\left(3.84 \times 10^{6}\right)$} \\
\hline Modulation type & \multicolumn{2}{|c|}{ QPSK } \\
\hline Mobile velocity & $400 \mathrm{~km} / \mathrm{h}$ & $800 \mathrm{~km} / \mathrm{h}$ \\
\hline $\begin{array}{l}\text { Normalized Doppler spread } \\
\left(f_{d} T_{s}\right)\end{array}$ & 0.0494 & 0.0988 \\
\hline
\end{tabular}

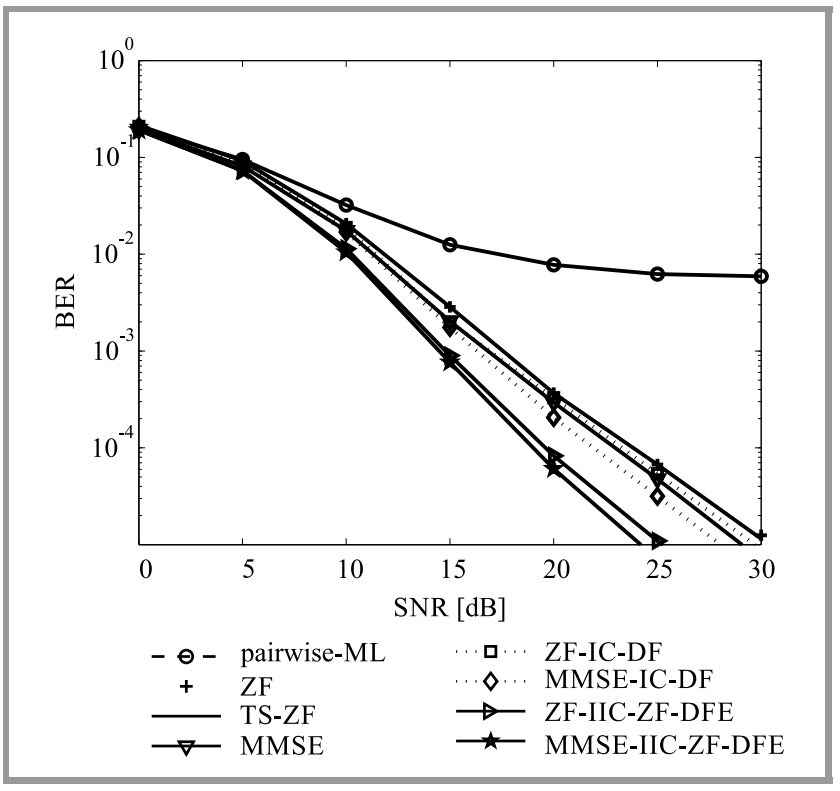

Fig. 2. BER performance comparison of various signal detection methods for QOSTBC at $f_{d} T_{s}=0.0494$.

BER performance of conventional and proposed detection methods for the correlation coefficient $f_{d} T_{s}=0.0494$ and 0.0998 are shown in Figs. 2 and 3, respectively. Simulation results show that the pairwise-ML method suffers

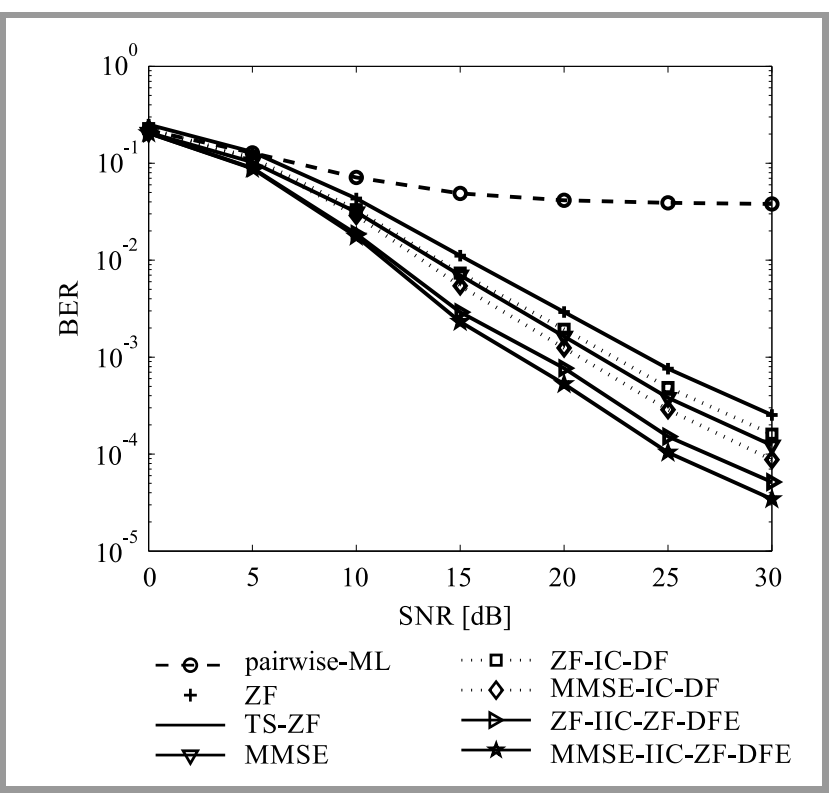

Fig. 3. BER performance comparison of various signal detection methods for QOSTBC system at $f_{d} T_{s}=0.0998$.

from severe performance degradation due to the time selective channel. ZF and TS-ZF, in turn, offer almost similar performance and outperform the pairwise-ML method, as both methods force the interferences signals to zero. The MMSE method gives better results than the ZF method, as it utilizes noise correlation properties. It offers almost the same performance as ZF-IC-DFE. MMSE-IC-DFE outperforms ZF-IC-DFE, as it uses the MMSE method instead of the ZF method to estimate the initial data symbol. MMSE-IC-DFE and ZF-IC-DFE offer better performance than their corresponding linear detection counterparts. However, both these methods suffer from performance degradation due to error floor, as less reliable data are propagated to estimate the other transmitted signal. Simulation results show that the proposed ZF-IIC-ZF-DFE method provides better results than the conventional MMSE-IC-DFE and ZF-IC-DFE methods, as more reliable data sequences are estimated in the iterative process using the ZF-DFE method. MMSE-IIC-ZF-DFE offers better results than ZFIIC-ZF-DFE, due to the implementation of MMSE rather than $\mathrm{ZF}$, in order to estimate the initial data information. Performance of different detection methods, in the descending order, is as follows: MMSE-IIC-ZF-DFE, ZF-IIC-ZFDFE, MMSE-IC-DFE, MMSE, ZF-IC-DFE, TS-ZF, ZF and pairwise-ML.

BER performance of the proposed and conventional signal detection methods, for different numbers of iterations (I) for $f_{d} T_{s}=0.0998$ at $25 \mathrm{~dB}$ SNR, is shown in Fig. 4. Simulation results show that the proposed ZF-IIC-ZF-DFE method performs significantly better than the ZF-IC-DFE method and slightly better than the MMSE-IC-DFE method for $I=2$. Performance increases even further as the number of iteration increases. MMSE-IIC-ZF-DFE has a much better performance than ZF-IIC-ZF-DFE. From the simulation, we observe that both proposed methods with $I=3$ 


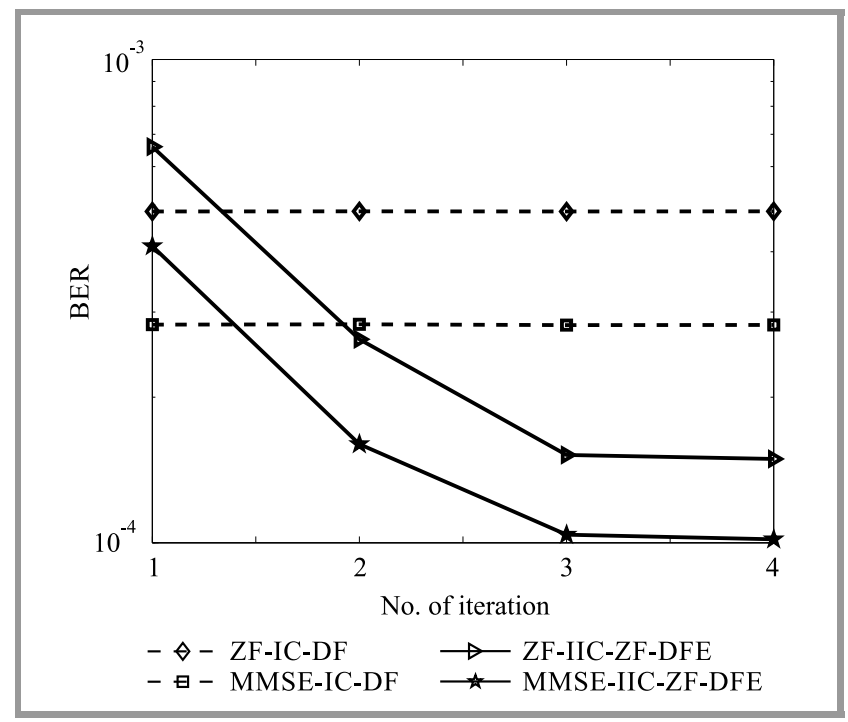

Fig. 4. BER versus number of iterations $I$ of various signal detection methods for QOSTBC system at $f_{d} T_{s}=0.0998$ at $25 \mathrm{~dB}$ SNR.

and $I=4$ offer nearly the same performance, and there is no further improvement as we go beyond $I=3$. Therefore, we assume $I=3$ for both these proposed methods.

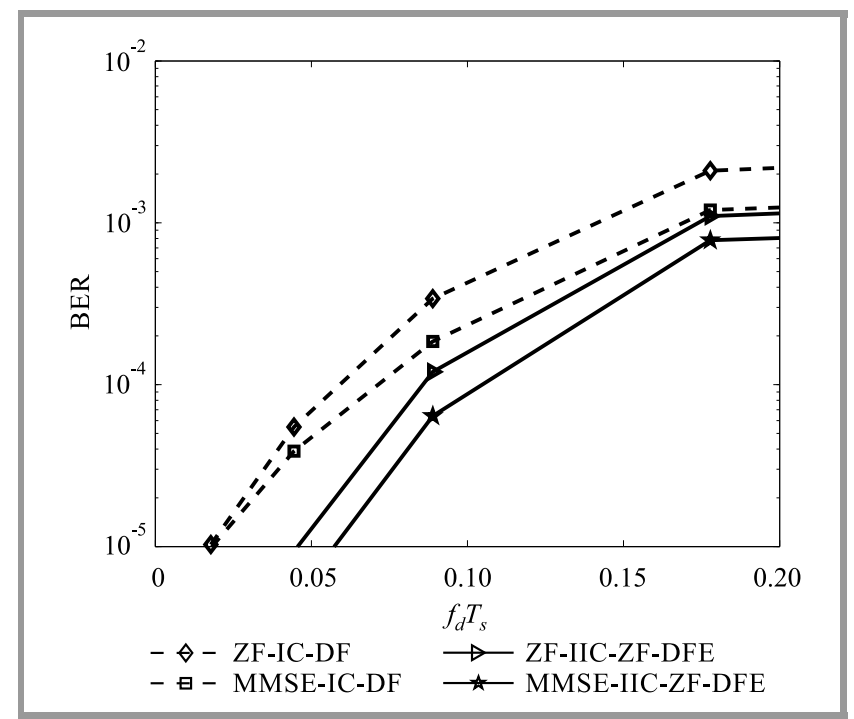

Fig. 5. BER versus $f_{d} T_{s}$ comparison of various signal detection methods for QOSTBC system at $25 \mathrm{~dB}$ SNR.

Figure 5 compares BER versus $f_{d} T_{s}$ performance of the proposed method and conventional ZF-IC-DFE and MMSEIC-DFE methods. Simulation results show that MMSE-ICDFE has a significantly performance than ZF-IC-DFE with the increase in Doppler spread. It may be noticed that, at very high $f_{d} T_{s}$ values, performance of the proposed ZFIIC-ZF-DFE method is slightly better than that of the conventional MMSE-IC-DFE method. Both proposed methods offer outstanding performance gains over the conventional methods, irrespective of the channel variation rate. Although the performance gap decreases as the Doppler frequency increases, MMSE-IIC-ZF-DFE still considerably outperforms the MMSE-IC-DFE method.

\section{Conclusions}

We have analyzed various conventional signal detection methods, e.g. ZF, TS-ZF, MMSE, ZF-IC-DFE and MMSEIC-DFE. Furthermore, we proposed two iterative signal detection methods, namely ZF-IIC-ZF-DFE and MMSE-IICZF-DFE to suppress interference effects for the QOSTBC system over a time selective channel. The simulation results show that both proposed methods significantly outperform the conventional methods in a high mobility channel environment.

\section{References}

[1] G. J. Foschini, "Layered space-time architecture for wireless communication in a fading environment when using multi-element antennas", Bell Labs Tech. J., vol. 1, no. 2, pp. 41-59, 1996 (doi: 10.1002/bltj.2015).

[2] H. Bolcskei, D. Gesbert, and A. J. Paulraj, "On the capacity of OFDM-based spatial multiplexing systems", IEEE Trans. on Commun., vol. 50, no. 2, pp. 225-234, 2002 (doi: 10.1109/26.983319).

[3] A. Paulraj, D. Gore, R. Nabar, and H. Bolcskei, "An overview of MIMO communications-a key to gigabit wireless", Proc. of the IEEE, vol. 92, no. 2, pp. 198-218, 2004 (doi: 10.1109/JPROC.2003.821915).

[4] W. Zhang, X.-G. Xia, and K. B. Letaief, "Space-time/frequency coding for MIMO-OFDM in next generation broadband wireless systems", IEEE Wirel. Commun., vol. 14, no. 3, pp. 32-43, 2007 (doi: 10.1109/MWC.2007.386610).

[5] S. M. Alamouti, "A simple transmit diversity technique for wireless communications", IEEE J. on Selec. Areas in Commun., vol. 16, no. 8, pp. 1451-1458, 1998 (doi: 10.1109/49.730453).

[6] O. Bejarano, E. W. Knightly, and M. Park, "IEEE 802.11ac: From channelization to multi-user MIMO", IEEE Commun. Mag., vol. 51, no. 10, pp. 84-90, 2013 (doi: 10.1109/MCOM.2013.6619570).

[7] "802.11ac: The Fifth generation of Wi-Fi technical white paper", Cisco, Tech. Rep., Aug. 2012 [Online]. Available: https://www.cisco.com/c/dam/en/us/products/collateral/wireless/ aironet-3600-series/white-paper-c11-713103.pdf

[8] Q. Li, G. Li, W. Lee, M.-i. Lee, D. Mazzarese, B. Clerckx, and Z. Li, "MIMO techniques in WiMAX and LTE: A feature overview", IEEE Commun. Mag., vol. 48, no. 5, pp. 86-92, 2010 (doi: 10.1109/MCOM.2010.5458368).

[9] V.-B. Pham, "Space-time block code design for LTE-advanced systems", Trans. on Emerg. Telecommun. Technol., vol. 26, no. 5, pp. 918-928, 2015 (doi: 10.1002/ett.2764).

[10] I. Eizmendi et al., "DVB-T2: The second generation of terrestrial digital video broadcasting system”, IEEE Trans. on Broadcast., vol. 60 , no. 2 , pp. $258-271,2014$ (doi: 10.1109/TBC.2014.2312811).

[11] D. Vargas, D. Gozalvez, D. Gomez-Barquero, and N. Cardona, "MIMO for DVB-NGH, the next generation mobile TV broadcasting", IEEE Commun. Mag., vol. 51, no. 7, pp. 130-137, 2013 (doi: 10.1109/MCOM.2013.6553689).

[12] V. Tarokh, H. Jafarkhani, and A. R. Calderbank, "Space-time block codes from orthogonal designs", IEEE Trans. on Inform. Theory, vol. 45, no. 5, pp. 1456-1467, 1999 (doi: 10.1109/18.771146).

[13] G. Ganesan and P. Stoica, "Space-time diversity using orthogonal and amicable orthogonal designs", in Proc. of IEEE Int. Conf. on Acoust., Speech, and Sig. Process. ICASSP 2000, Istanbul, Turkey, 2000, pp. 2561-2564 (doi: 10.1109/ICASSP.2000.860978).

[14] C. Papadias and G. Foschini, "Capacity-approaching space-time codes for systems employing four transmitter antennas", IEEE Trans. on Inform. Theory, vol. 49, no. 3, pp. 726-733, 2003 (doi: 10.1109/TIT.2002.808119). 
[15] H. Jafarkhani, "A quasi-orthogonal space-time block code", IEEE Trans. on Commun., vol. 49, no. 1, pp. 1-4, 2001 (doi: 10.1109/26.898239).

[16] M. Rupp and C. Mecklenbrauker, "On extended Alamouti schemes for space-time coding", in Proc. of IEEE 5th Int. Symp. on Wirel. Pers. Multimed. Commun. WPMC 2002, Honolulu, HI, USA, 2002, pp. 115-119 (doi: 10.1109/WPMC.2002.1088143).

[17] M. Gao, L. Zhang, C.-1. Han, and J.-h. Ge, "Low-complexity detection schemes for QOSTBC with four-transmit-antenna", IEEE Commun. Lett., vol. 19, no. 6, pp. 1053-1056, 2015 (doi: 10.1109/LCOMM.2015.2417883).

[18] M. Towliat and H. Zamiri-Jafarian, "Low-complexity QRD-based detection scheme for full rate QOSTBC with four-transmit antenna", in 6th Int. Symp. on Telecommun. IST 2012), Tehran, Iran, 2012 (doi: 10.1109/ISTEL.2012.6482969).

[19] M.-T. Le, V.-S. Pham, L. Mai, and G. Yoon, "Low-complexity maximum-likelihood decoder for four-transmit antenna quasi-orthogonal space-time block code", IEEE Trans. on Commun., vol. 53, no. 11, pp. 1817-1821, 2005 (doi: 10.1109/TCOMM.2005.858688).

[20] E. Nassaji, M. Ahmadi Almasi, and M. Fardad, "Low-complexity decoder based on conditional ML for QOSTBC", IEEE Commun. Lett., vol. 22, no. 8, pp. 1548-1551, 2018 (doi: 10.1109/LCOMM.2018.2828825).

[21] Y. Zhang and H. Liu, "Impact of time-selective fading on the performance of quasi-orthogonal space-time-coded OFDM systems", IEEE Trans. on Commun., vol. 54, no. 2, pp. 251-260, 2006 (doi: 10.1109/TCOMM.2005.863773).

[22] Y. Wang, J. Jin, and Y. Park, "Performance of quasi-orthogonal space-time block code over time-selective channel with linear and decision feedback equalizations", in Proc. IEEE 8th Int. Con. Adv. Commun. Technol. ICACT 2006, Phoenix Park, South Korea, 2006, pp. 1095-1100 (doi: 10.1109/ICACT.2006.206163).

[23] Y. Wang, J. Wang, L. Zhaobiao, and X. Liu, "Performance improvement of QO-STBC over time-selective channel for wireless network", J. of Netw. and Comp. Appl., vol. 36, no. 3, pp. 1018-1026, 2013 (doi: 10.1016/j.jnca.2012.01.005).

[24] F.-C. Zheng and A. G. Burr, "Signal detection for non-orthogonal space-time block coding over time-selective fading channels", IEEE Commun. Lett., vol. 8, no. 8, pp. 491-493, 2004 (doi: 10.1109/LCOMM.2004.832753).

[25] F.-C. Zheng and A. G. Burr , "Signal detection for orthogonal spacetime block coding over time-selective fading channels: A PIC approach for the G/sub i/systems", IEEE Trans. on Commun., vol. 53, no. 6, pp. 969-972, 2005 (doi: 10.1109/TCOMM.2005.849990).

[26] L.-Y. Song and A. G. Burr, "Successive interference cancelation for space-time block codes over time-selective channels", IEEE Commun. Lett., vol. 10, no. 12, pp. 837-839, 2006 (doi: 10.1109/LCOMM.2006.060993).

[27] Y. R. Zheng and C. Xiao, "Simulation models with correct statistical properties for Rayleigh fading channels", IEEE Trans. on Commun., vol. 51, no. 6, pp. 920-928, 2003 (doi: 10.1109/TCOMM.2003.813259).

[28] J. G. Proakis, Digital Communication, 4th ed. McGraw-Hill, 2001 (ISBN: 9780072321111).

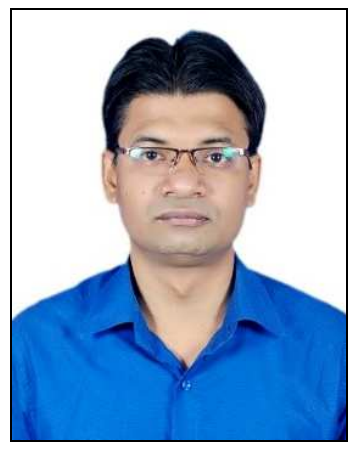

Jyoti Prasanna Patra received his Ph.D. and M.Tech. degrees from the National Institute of Technology Rourkela, India in 2018 and 2012, respectively. $\mathrm{He}$ has completed his B.Tech. degree from Biju Patnaik University of Technology, Odisha, India in 2008. Currently, he is working as a senior Assistant Professor at the Department of Electronics and Communication Engineering of the Madanapalle Institute of Technology and Science, Madanapalle, Andhra Pradesh, India. His research interests include signal processing for wireless communication.

E-mail: jyotiprasannap@mits.ac.in

Department of Electronics and Communication

Engineering

Madanapalle Institute of Technology and Science

Madanapalle

Andhra Pradesh-517325, India.

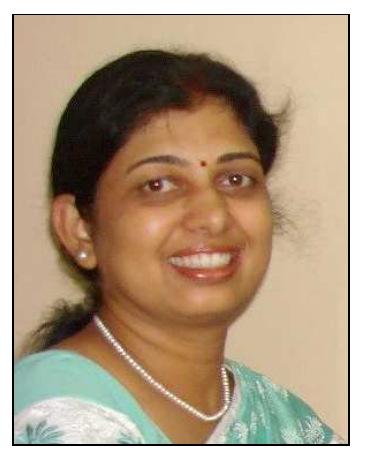

Poonam Singh received her B.Tech. degree from University College of Engineering Burla, Odisha, India in 1988, and M.Tech. degree from Regional Engineering College, Rourkela, India in 1991. She completed her Ph.D. at the Indian Institute of Technology, Kharagpur, India. She has been a faculty chair at the National Institute of Technology, Rourkela (formerly known as Regional Engineering College) since 1991. She is presently an Associate Professor at the National Institute of Technology, Rourkela, India. She is a senior member of IEEE and a life member of ISTE. Her teaching and research interests include analog and digital communications, wireless communication systems, spread spectrum modulation, multicarrier systems, etc.

E-mail: psingh@ @itrkl.ac.in

Department of Electronics and Communication

Engineering

National Institute of Technology

Rourkela

Odisha-769008, India 